\title{
CORRECTION
}

\section{Correction: IQGAP1 promotes anoikis resistance and metastasis through Rac1-dependent ROS accumulation and activation of Src/FAK signalling in hepatocellular carcinoma}

Chun-fen Mo, Jun Li, Shu-xia Yang, Hui-jie Guo, Yang Liu, Xing-yan Luo, Yan-tang Wang, Min-hui Li, Jing-yi Li and Qiang Zou

British Journal of Cancer (2021) 125:622; https://doi.org/10.1038/s41416-021-01449-z

Correction to: British Journal of Cancer https://doi.org/10.1038/ s41416-020-0970-z, published online 7 July 2020

The original version of this article unfortunately contained a mistake in the funding information. During their recent project check, the authors found that due to their negligence, the project National Natural Science Foundation of China, No. 81702446 was not included in the "funding information" section of their published article. The authors apologize for the mistake. The correct funding information should read: This study was supported by the National Natural Science Foundation of China (81402944, 81702446, 81871300), Application and Basic Project of Science and Technology Department of Sichuan Province (2018JY0440, 2017JY0174) and Research Fund of Chengdu Medical College (CYZ16-01). The original article has been revised. 